\title{
Particle tracking analysis of extracellular vesicles as a non- destructive surrogate tool for mesenchymal stromal cell health monitoring
}

\author{
Gary M Morley", Dorota Bartczak*,, Heidi Goenaga-Infante and Jonathan J Campbell \\ LGC Limited, Queens Road, Teddington, TW11 0LY, UK \\ \#These authors have contributed equally to the experimental work
}

\begin{abstract}
Mesenchymal Stromal Cells (MSCs) have become a promising candidate for autologous cell therapy for a number of human pathologies, most notably in the treatment of auto-immune diseases. However, to achieve effective clinical dosing, relatively large numbers of these cells are required, making extensive in vitro expansion paramount. This poses an increased risk of contamination, starvation or over-expansion of cells during the cultivation process, likely affecting their integrity and quality. To prevent this from happening more efficient monitoring systems are urgently needed. Here we have tested a simple and non-destructive approach based on the Particle Tracking Analysis (PTA) platform for in-line monitoring of MSC health during expansion. The proposed approach relies on the surrogate measurements of MSC health through size analysis of extracellular vesicles (EVs) released by these cells into their microenvironment. We have found that a range of growth stressors, such as over-expansion, serum depletion (mimicking starvation) or trace amounts of Lipopolysaccharide (simulating bacterial contamination) all result in perturbations in the $\mathrm{EV}$ profiles as well as significant changes in their modal and/or mean diameter values.
\end{abstract}

\section{Introduction}

Mesenchymal Stromal Cells (MSCs) continue to generate much interest as autologous cell therapy products owing to high efficiency in wound and tissue repair [1] and unique immune-modulation abilities [2]. MSCs can either heighten an immune response when pathogens are present or reduce it when a heightened immune response is deleterious, such as in case of sterile inflammation [2]. Such properties have led many to refer to these cells as "guardians of inflammation" [2]. In addition to this, MSC have been shown to reduce T-cell responses [3], which along with a lack of class II and low levels of class I selfrecognition markers, [4] allows them to temporarily evade immune recognition. Such properties enable their applications as cell therapy products, provided these relatively rare cells can be expanded to suitable numbers in vitro to meet dosing requirements, with 1-2 million cells per kilogram of the recipient being the most commonly used number at present [5].

The need for extensive in vitro expansion during bioprocessing can prolong exposure to non-optimal biochemical and biophysical cues in the culture microenvironment, resulting in a range of undesired effects. In fact, the failure of at least one phase III clinical trial has been attributed to the over-expansion of these cells and the associated loss of quality [5]. The main issue seems to be the lack of clear guidance as to what represents the maximal expansion MSCs can undergo before losing their beneficial abilities. So far only one clinical trial has compared early and late passage cells with the conclusion that early cells were more efficacious than the older, late passage cells [6]. There is therefore a clear need for the development of effective quality monitoring systems for these cells in culture, which could circumvent the uncertainty associated with traditional labelling technologies, and more importantly abrogate the need for direct cell sampling strategies, which can introduce contamination of these high value products $[7,8]$.
Here, we propose an attractive alternative approach for the assessment of health of MSCs in culture based on the analysis of size profiles of extracellular vesicles (EVs) secreted by the MSCs. EVs typically comprise $50-150 \mathrm{~nm}$ exosomes, $100-400 \mathrm{~nm}$ ectosomes and up to $1000 \mathrm{~nm}$ apoptotic bodies and have been shown to possess important characteristics of the parent cells [9]. In fact, it has been demonstrated that depending on the amount of nutrients in the media or the level of exhaustion of the cells, biochemical composition of the secreted EVs might vary [10]. We demonstrate here for the first time, that the size characteristics of the secreted EVs also reflect the health of the parent cells exposed to extreme conditions in culture. Particle Tracking Analysis (PTA) platform, which is well suited for size measurements of polydispersed and multimodal samples in suspension such as EVs [11], being a number-based [12], rather than an ensemble technique like e.g. Dynamic Light Scattering [13], was used in this study to monitor the MSC derived EVs. EVs showed significant changes in their size distribution profiles as well as modal and/or mean diameter values upon the adverse culture conditions tested here, such as the lack of nutrients (reduced supplementation with serum), bacterial contamination (added Lipopolysaccharide, 'LPS') and over-expansion. Thus PTA profiling of the EVs demonstrated here has potential to provide a basis for the future development of in-/at-line monitoring strategies in the field of cell therapies.

${ }^{*}$ Correspondence to: Bartczak D, LGC Limited, Queens Road, Teddington, TW11 0LY, UK, E-mail: dorota.bartczak@lgcgroup.com

Jonathan J Campbell, LGC Limited, Queens Road, Teddington, TW11 0LY, UK, E-mail: jonathan.campbell@lgcgroup.com

Received: April 11, 2019; Accepted: April 22, 2019; Published: April 25, 2019 


\section{Materials and Methods}

\section{Cell culture}

Three bone marrow MSC lines were obtained from commercial suppliers (Rooster Bio cat. MSC-001 'MSC1', ScienCell cat. 7500 'MSC2' and PromoCell cat.C-12974 'MSC3'). Master stocks were generated in the suppliers recommended media following the supplied protocol for each line. For all experiments the cells were incubated in a humidified incubator at $5 \% \mathrm{CO} 2,37^{\circ} \mathrm{C}$. The cells were passaged in vitro in a common media, D10 (DMEM, Gibco, cat. 41965-039 containing $10 \%$ FBS, Gibco, cat. 16000-044) following an identical passaging and banking protocol. The cells were expanded for at least one passage (7 days) in D10 prior to commencement of experiments. The cells were seeded into T175 flasks at 4'000 cells per $\mathrm{cm}^{2}$ and grown for 7 days per passage with media replenished 2-3 times per week. Aliquots of cells were banked at each passage for later analysis. Expansion was stopped when the doubling time of the cells approached 5 days. The exception to this was the MSC2 line which showed a high doubling time even at the first analysis passage (Table S1).

For each MSC line (MSC1, MSC2 and MSC3) 3 separate passages were removed from liquid nitrogen storage one passage prior to the desired target passage. The cells were revived for 5 days as described above before transferring into 6-well plates (in triplicate) at $20^{\prime} 000$ cells per $\mathrm{cm}^{2}$ with $0.3 \mathrm{~mL} / \mathrm{cm}^{2}$ D10 per well. Excess cells from each line and passage were also used to seed 24-well plates for senescent cell detection. After resting for 24 hours the media was removed and the cells washed $3 \mathrm{x}$ with $4 \mathrm{~mL}$ PBS (Gibco cat. D8537) per well prior to addition of exosome depleted D10 (exo-D10, DMEM, Gibco, cat. 41965-039 containing 10\% exosome depleted FBS, Gibco, cat. A2720803) at a volume of $0.2 \mathrm{~mL} / \mathrm{cm}^{2}$. The exception to this was for serum depletion experiments. Identical media volumes were also added to empty wells (without any cells). The cells and media were placed in the incubator, prior to collecting after 24 hours and processing according to the EVs harvest and purification protocol.

Experiments with varied LPS and FBS content added to the cells were performed in triplicate on MSC1 line at the early passage 'E' level. The experiments were performed on three different days using different expansions of cell from banked stocks one passage before desired, as described above. After seeding at $20^{\prime} 000$ cells per $\mathrm{cm}^{2}$ in to $0.3 \mathrm{~mL} / \mathrm{cm}^{2} \mathrm{D} 10$ in 6 -well plates and resting for 24 hours the media was removed and the cells were washed $3 \mathrm{x}$ with PBS. LPS (SigmaAldrich cat. L4391) at a concentration varied from $0 \mathrm{pg} / \mathrm{mL}$ to $5 \mu \mathrm{g} /$ $\mathrm{mL}$ was prepared in exosome depleted D10 media and then added to the cells at a final volume of $0.2 \mathrm{~mL} / \mathrm{cm}^{2}$. After 24 hours the media was collected and processed according to the EVs harvest and purification protocol. For FBS titrations an identical procedure was followed with the exception that the starting $10 \%$ exosome depleted media was used and serial dilutions set up in DMEM media (no exogenous FBS).

\section{Determination of cell senescence levels}

Senescent cells were detected using a commercial kit (Senescence Cells Histochemical Staining Kit, Sigma-Aldrich, cat. CS0030) following the supplied protocol for that kit. Briefly, excess cells from each line and passage were transferred in triplicate to 24 -well plates at a density of 1'000 cells per $\mathrm{cm}^{2}$ in $0.3 \mathrm{~mL}$ of D10 per well. After 24 hours the media was replaced by a further $0.3 \mathrm{~mL}$ of D10 per well and the cells returned to the incubator for a further day. After this time the media was removed, the cells washed $1 x$ PBS before fixing the cells in $1 x$ fixative (supplied with the kit) for $7 \mathrm{~min}$ at room temperature. The fixative was then removed, the cells washed $3 \mathrm{x}$ with $\mathrm{PBS}$ before the addition of $\beta$-galactosidase staining solution. The cells were incubated at $37^{\circ} \mathrm{C}$ overnight in ambient $\mathrm{CO} 2$ levels. The next day the stain was removed and the wells rinsed with PBS before adding $70 \%$ glycerol to the wells (v/v glycerol, Sigma-Aldrich, cat. G5516) and storing the plates at $4{ }^{\circ} \mathrm{C}$ until imaging. Images were taken from each of the triplicate wells at 10x magnification using a Palm LCM Zeiss (27 images per condition) and the number of total and stained blue (senescent) cells manually counted.

\section{EV harvest and purification}

Collected cell culture media was transferred to plastic tubes (Eppendorf Protein LoBind, Fisher Scientific cat. E925000092) and centrifuged at 2'000 g for $30 \mathrm{~min}$ in a Picol7 centrifuge to remove cell debris. Around $900 \mu \mathrm{L}$ of sample was then transferred to a fresh tube and $450 \mu \mathrm{L}$ of Exosome Isolation Reagent (Invitrogen 4478359 lot. 0054664) was added. The tubes were inverted before vortexing for 3-5 seconds and placing overnight at $4{ }^{\circ} \mathrm{C}$. The following day the samples were transferred to a Jouan BRi4 centrifuge and centrifuged at $10^{\prime} 000 \mathrm{~g}$ for 1 hour at $4{ }^{\circ} \mathrm{C}$. The supernatant was then removed and $700 \mu \mathrm{L}$ of 0.2 $\mu \mathrm{m}$ filtered PBS (D8537 lot. RNBG4118) was added per tube and the tubes stored at $-80^{\circ} \mathrm{C}$ until PTA analysis.

\section{PTA analysis of the EVs}

PTA measurements and analysis of the recorded movies were performed with the NanoSight NS500 model, manufactured by Malvern Panalytical and equipped with a violet diode laser light source (405 nm CW, max power $<60 \mathrm{~mW}$ ), an EMCCD camera and NTA3.2 software. The instrument was thoroughly cleaned at the beginning of each day and the number of particles seen in the blank sample $(0.2$ $\mu \mathrm{m}$ filtered PBS used as dispersant for MSCs EVs) was determined prior to analysis of the MSCs EVs samples. Temperature was set and maintained at $25^{\circ} \mathrm{C}$ throughout the analysis. Camera levels were set by the user and kept the same for all samples, focus levels were adjusted accordingly on each day of the analysis. Each aliquot was measured at least five times under repeatability conditions with a fresh portion of the sample loaded before each 60s long measurement. Detection threshold was set by the user to 5 and kept constant throughout the analysis. Alongside EVs samples, polystyrene particles from Thermo Scientific (3100A, nominal diameter of $100 \mathrm{~nm}$ ) were measured for quality control purposes. Around 50-100 particles per field of view were seen depending on the sample, resulting in a minimum of $\sim 2$ '000 completed tracks per video. Data shown represent mean $+/$ - standard deviation from all replicate measurements.

\section{Results}

Three commercially available MSCs lines were chosen for this study. In the first instance we assessed the consistency in the mean and modal diameter values as well as the size distribution profiles of EVs produced by the MSC1 line grown under normal conditions (early passage 'E', endotoxin and antibiotic free exosome depleted DMEM media supplemented with $10 \%$ exosome depleted FBS, grown for 24 hours at $37^{\circ} \mathrm{C}$ in a humidified air balanced with $5 \% \mathrm{CO}_{2}$ incubator). A total of 9 independent experiments were performed on MSC1E over the duration of 24 months and using different expansions of the cells from masterbank stocks. Purified MSC1E EVs were examined alongside EVs derived from cell culture media only (no cells in the well) analysed on 11 separate occasions. PTA measurements of mean and mode EVs diameters derived from MSC1E and FBS (Figure 1) were highly reproducible, with the relative standard deviation (RSD) of better than 
$10 \%$ for the mode and $15 \%$ for the mean achieved. Statistical analysis with the student $\mathrm{t}$-test showed that EVs derived from MSC1E were significantly different in their mode and mean sizes from the EVs of bovine origin (Table 1), with an average modal diameter $110.9 \mathrm{~nm}$ and mean of $180.9 \mathrm{~nm}$.

\section{Effect of MSC age}

Size characteristics of the EVs secreted by the cells of different passage number, were then compared against the level of senescence (Table 2). Small changes were observed in the size distribution profiles of the three tested MSC EV samples that tended towards an observed increase in EV size with increasing passage. This was reflected by observed changes in the mean and modal diameters (Figure 2B), with EVs secreted by the early passage cells being smaller overall than EVs from late passage cells. Although this trend can be seen in all tested scenarios, a statistically significant change was seen for modal diameter in case of MSC1 line and mean diameter for MSC2 and MSC3 lines. This seems to be correlated with the level of cell senescence (Figure 2C) showing only up to $19.9 \%$ for the MSC1 line at the late passage, whilst over $40 \%$ for MSC2 and MSC3 lines. For relatively young cells modal EVs diameter, representing the most abundant size fraction seems a better measure of age-triggered changes than mean size representing average size of all the sub-fractions of EVs present.

\section{Effect of MSC exposure to varied LPS levels}

MSCs are known for their ability to modulate the response of the immune system through various triggers. For example, they can respond to bacterial endotoxin such as LPS via the Toll-like-receptor 4 (TLR4) and this epitope engagement can switch these cells from an anti-inflammatory to an inflammatory state [14]. We therefore examined the MSCs response to this endotoxin, by measuring the EVs

Table 1. PTA analysis of MSC and FBS derived EVs ( $n=9$ for MSC and $n=11$ for FBS).

\begin{tabular}{|c|c|c|c|c|c|}
\hline \multirow{2}{*}{ Parameter } & \multirow{2}{*}{ EV origin } & \multirow{2}{*}{$\begin{array}{c}\text { Average } \\
(\mathbf{n m})\end{array}$} & SD & RSD & \multirow{2}{*}{$\mathbf{p}^{*}$} \\
\hline & & & (nm) & $(\%)$ & \\
\hline \multirow{2}{*}{$\begin{array}{l}\text { Mode } \\
\text { diameter }\end{array}$} & MSC & 110.9 & 5.7 & 5.1 & \multirow{2}{*}{$<0.001$} \\
\hline & FBS & 87.5 & 8.7 & 10 & \\
\hline \multirow{2}{*}{$\begin{array}{l}\text { Mean } \\
\text { diameter }\end{array}$} & MSC & 180.9 & 21.3 & 11.8 & \multirow{2}{*}{$<0.001$} \\
\hline & FBS & 135.5 & 18.4 & 13.6 & \\
\hline
\end{tabular}

*Calculated with student t-test

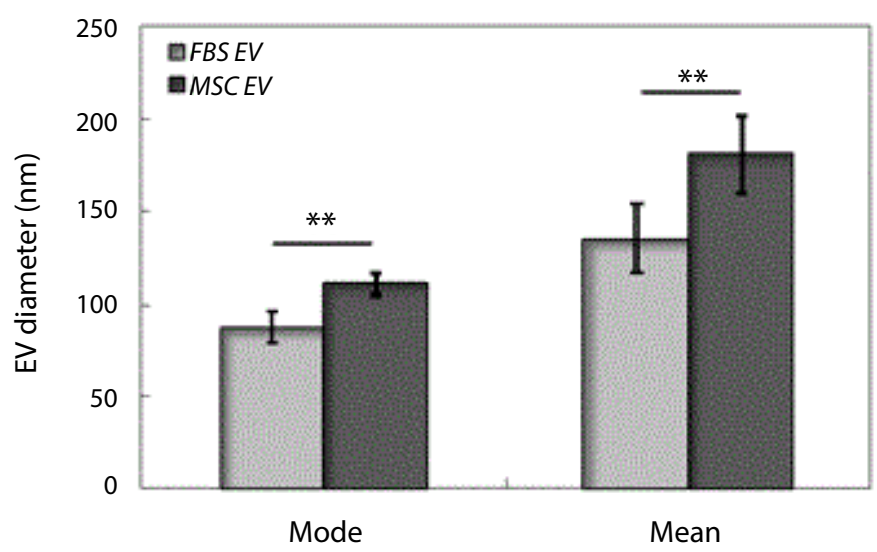

Figure 1. PTA analysis of MSC and FBS derived EVs show significant difference. Mean and modal diameters of EV derived from MSC1E and FBS are shown. Data represent average \pm SD from 9 (for MSC) or 11 (for FBS) independent experiments. Significance levels were assessed with student t-test, $p<0.001$ is marked with ' $* *$ ' indicating statistically very significant data sets
Table 2. PTA analysis of MSC derived EVs (average \pm SD, n=3) and the associated senescence levels at early and late cell passage

\begin{tabular}{|l|c|c|c|c|}
\hline \multirow{2}{*}{ Cell line } & $\begin{array}{c}\text { Relative cell } \\
\text { passage }\end{array}$ & $\begin{array}{c}\text { Modal EV } \\
\text { diameter }(\mathbf{n m})\end{array}$ & $\begin{array}{c}\text { Mean EV } \\
\text { diameter }(\mathbf{n m})\end{array}$ & Senesce level \\
\hline \multirow{2}{*}{ MSC1 } & early & $104.3 \pm 0.7$ & $159.8 \pm 10.2$ & $7.1 \pm 3.0$ \\
\hline \multirow{2}{*}{ MSC2 } & late & $116.2 \pm 9.4$ & $165.9 \pm 12.5$ & $19.9 \pm 4.4$ \\
\hline \multirow{2}{*}{ MSC3 } & early & $110.9 \pm 4.4$ & $167.3 \pm 10.2$ & $19.9 \pm 2.7$ \\
\cline { 2 - 5 } & late & $115.8 \pm 5.9$ & $204.6 \pm 17.1$ & $46.7 \pm 12.1$ \\
\hline & early & $111.7 \pm 7.3$ & $163.7 \pm 12.5$ & $3.9 \pm 2.0$ \\
\cline { 2 - 5 } & late & $119.9 \pm 3.8$ & $208.4 \pm 33.5$ & $40.7 \pm 10.2$ \\
\hline
\end{tabular}

sizes and size profiles with PTA. LPS was added to cell culture at varied levels, starting from the amounts at which no contamination would be visible by the naked eye (i.e. turbid appearance of the media) and even below levels allowed to be present in endotoxin-tested sterile water, up to levels representing media contaminated with relatively high levels of bacterial contamination, exceeding those already visible by the naked eye. MSC1E cells treated with modest levels of LPS (10 pg/mL - 100 $\mathrm{ng} / \mathrm{mL}$ ) for 24 hours already showed a distinct shift in the PTA size profiles (Fig. 3 top), corresponding to a significant increase in the modal diameter values from $116.8 \pm 0.4 \mathrm{~nm}$ (average $\pm S D, n=3$ ) for $0 \mathrm{pg} / \mathrm{mL}$ LPS to $141.6 \pm 4.0 \mathrm{~nm}$ (average $\pm \mathrm{SD}, \mathrm{n}=3$ ) for $100 \mathrm{pg} / \mathrm{mL}$ LPS and $141.9 \pm 4.4 \mathrm{~nm}$ (average $\pm \mathrm{SD}, \mathrm{n}=3$ ) for $100 \mathrm{ng} / \mathrm{mL}$ LPS (Fig. 3 bottom). Although, not statistically significant, even at the lowest tested dose of LPS $(10 \mathrm{pg} / \mathrm{mL})$, an upwards trend in the EVs modal diameter can already be seen (to $123.8 \pm 3.0$, average $\pm S D, n=3$ ). At the highest tested dose $(250 \mathrm{ng} / \mathrm{mL}$ and $1 \mu \mathrm{g} / \mathrm{mL})$ the modal sizes and size distribution profiles reverted to the baseline levels. Although there was no significant change in the EVs at any dose, a similar trend to that of the mode was observed (Figures S2 and S3).

\section{Effect of serum depletion}

We next mimicked a sub-optimal feeding regime by exposing MSC1E cells to declining levels of FBS in culture media, from between $10 \%$ (typical) to $0 \%$ final concentration for 24 hour duration (Figure 4 ). There was a change in the EVs size profiles (Figure 4 top) and a gradual increase in the EVs modal diameter values as the serum levels declined (Figure 4 bottom). Statistically significant changes from $111.6 \pm 3.6 \mathrm{~nm}$ (average $\pm S D, n=3$ ) measured for $10 \%$ FBS supplementation were seen at all tested serum depletion levels, with EVs diameter increasing to $123.7 \pm 0.7 \mathrm{~nm}$ for $5 \% \mathrm{FBS}, 129.3 \pm 5.3 \mathrm{~nm}$ (average $\pm \mathrm{SD}, \mathrm{n}=3$ ) for $2 \%$, $137.4 \pm 7.8 \mathrm{~nm}$ (average $\pm \mathrm{SD}, \mathrm{n}=3$ ) for $1 \%$ and finally $132.5 \pm 7.2 \mathrm{~nm}$ (average $\pm S D, n=3$ ) for $0 \%$ FBS present. In a similar fashion to the LPS titration, there was no significant changes to the EVs mean diameter values in response to FBS depletion.

\section{Discussion}

We have demonstrated that size distribution profiles of EVs measured with PTA are fairly consistent and representative of the health of cultured cells, such as MSCs, providing the cell seeding numbers and the volume of added cell culture media were constant. With RSD ( $n=9$ for MSC EV and $n=11$ for FBS EV) better than $10 \%$ for the modal and $15 \%$ for mean diameters, significant differences between the MSCs and FBS derived EVs were seen (Fig. 1) with both, mean and modal diameter of the bovine EVs being much smaller than those of MSCs derived EVs and clearly distinguishable with PTA. This is of critical importance when interpreting the experimental data. Even when exosome-depleted media is used throughout, such media still contains some level of FBS derived EVs, with only about $90 \%$ depletion guaranteed by the manufacturer of the media used in the research presented here. 
A

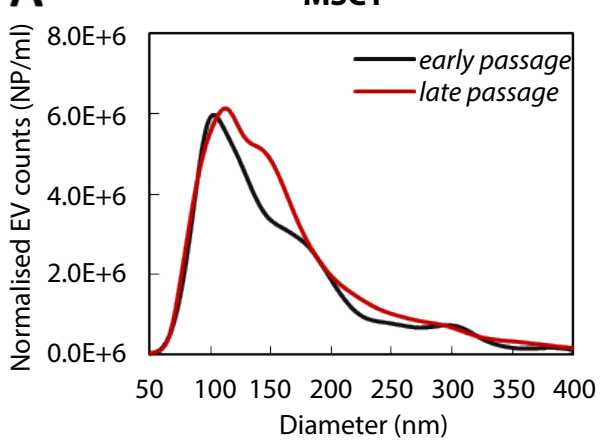

B PTA mean diameter

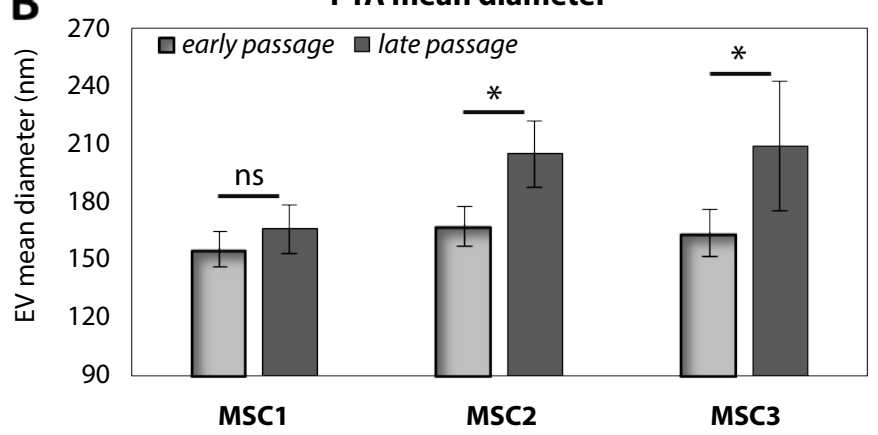

C

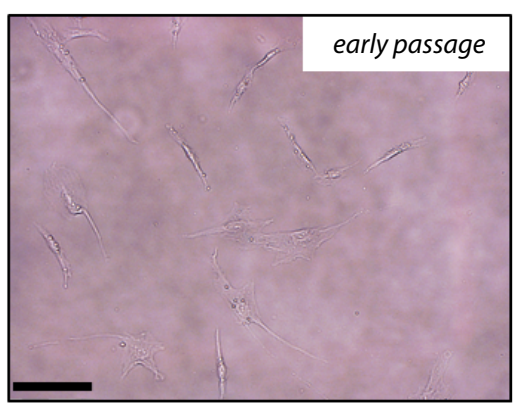

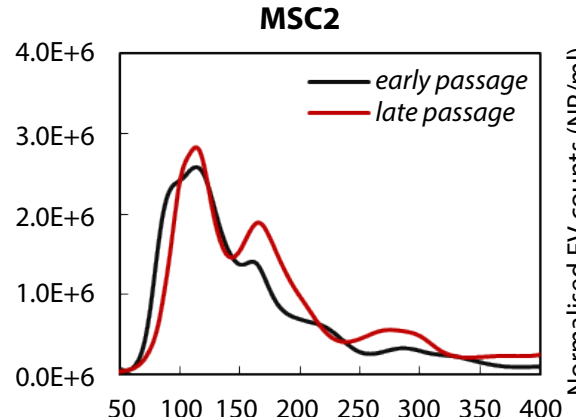

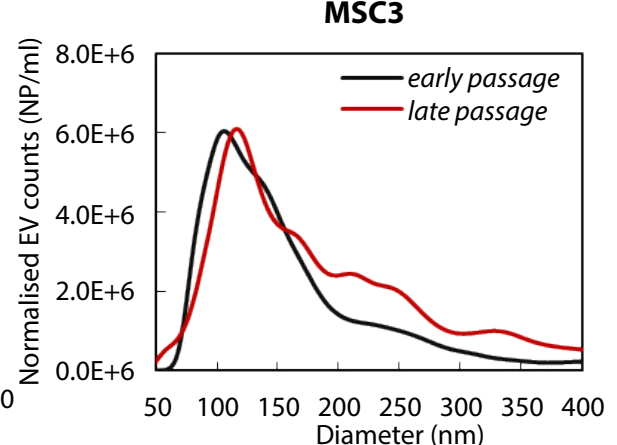

PTA modal diameter

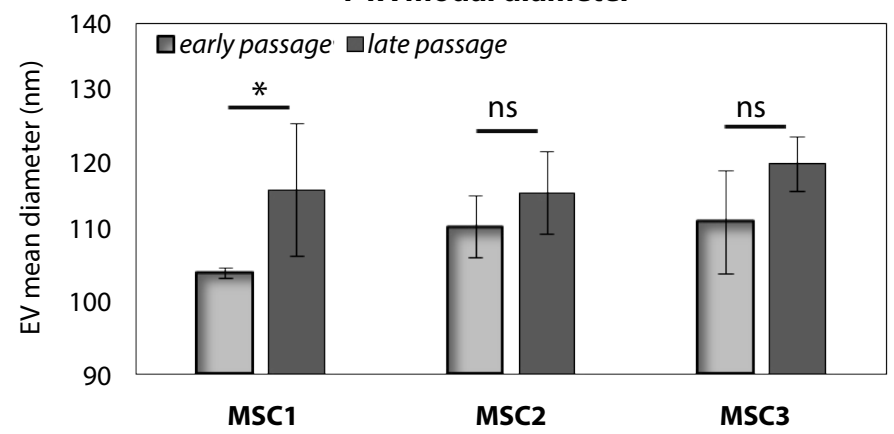

MSC1

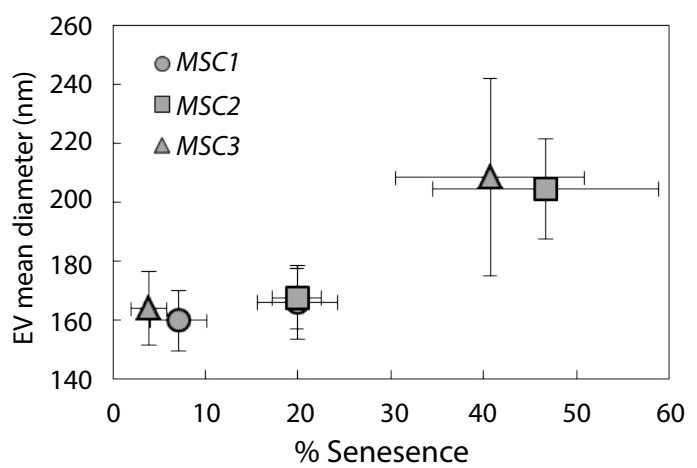

Figure 2. MSCs age effect on EVs profiles. EVs profile changes between early (black line) and later passage (red line) cells measured with PTA (A). PTA mean and the modal diameter of the EVs secreted by cells in the late and earlier passages shown as average $\pm \mathrm{SD}, \mathrm{n}=3$ and significance (student $\mathrm{t}$-test) marked with '*' for $\mathrm{p}<0.05$ and 'ns' for $\mathrm{p} \geq$ 0.05 (B). Representative images or early and late passage cells, where the senescent cells (arrows) are stained blue with $\beta$-galactosidase, scale bars are $150 \mu \mathrm{m}$ and correlation between the senescence levels of the cells and the EVs mean diameter shown as average $\pm \mathrm{SD}, \mathrm{n}=3$ (C)
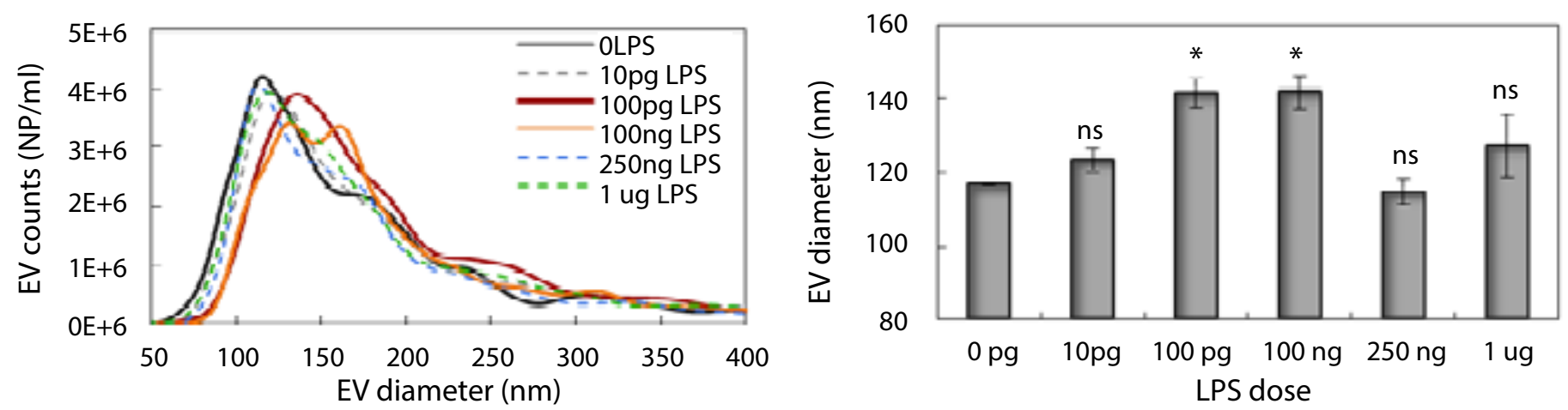

Figure 3. LPS dose effect on EVs profiles. Changes in the EVs profiles measured with PTA in response to LPS between 0 pg and $1 \mu \mathrm{g}$ added into the MSCs culture (top). PTA modal diameter of the EVs secreted by the cells shown as average $\pm \mathrm{SD}, \mathrm{n}=3$ and significance (student t-test) marked with '*' for $\mathrm{p}<0.05$ and 'ns' for $\mathrm{p} \geq 0.05$ measured against 0 pg LPS control (bottom) 

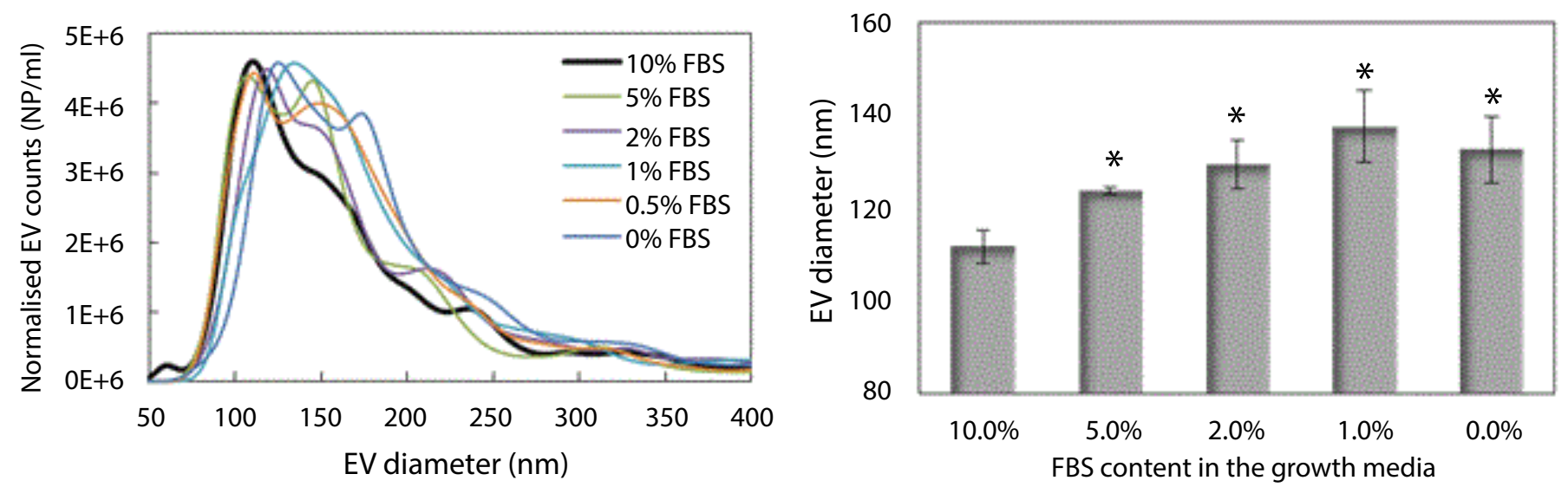

Figure 4. FBS content effect on EVs profiles. Changes in the EVs profiles measured with PTA in response to varied between 0 and $10 \%$ FBS content in the MSCs cell growth media, with $10 \%$ FBS control shown as thick line (top). PTA modal diameter of the EVs secreted by the cells shown as average \pm SD, $\mathrm{n}=3$ and significance (student $\mathrm{t}$-test) marked with '*' for $\mathrm{p}<0.05$ and 'ns' for $\mathrm{p} \geq 0.05$ measured against $10 \%$ FBS control (bottom)

The health of cultured cells can be affected by many factors, the most common being bacterial contamination, insufficient amount of nutrients and over-expansion. We have tested the impact of all these extreme conditions on the profiles of EVs secreted by the MSCs and have found significant changes in their size distribution profiles from the EVs derived from cells grown in the optimal environment. Broadening of the EVs size distribution profiles in conjunction with multimodal appearance occurred as the cells progressed in culture from early to late passage, concurrent with increased levels of cellular senescence (Figure 2). This corresponded with a significant increase in the EVs modal diameters and could be a good measure of overexpansion, which has been linked with the failure of at least one clinical trial featuring MSCs [5]. An increase in the modal EVs diameter was also seen as a result of MSCs response to LPS. Although, correlation between the bacterial endotoxin levels, such as LPS, and the number of bacteria present is quite variable, depending on the type of bacteria as well as the phase of bacterial growth, it can be approximated that $100 \mathrm{pg} / \mathrm{mL}$ LPS added to cell media is roughly 1'000-fold less than the level of contamination detectable by a naked eye (around $1.5 \times 108$ colony forming units per $\mathrm{mL}$ or 0.5 on the McFarlane scale)[15]. Even at these trace amounts of LPS $(100 \mathrm{pg} / \mathrm{ml})$, the effect on the profiles of MSCs derived EVs was significant, with an increase of around $25 \mathrm{~nm}$ of the mode size above that of the MSC1E grown in optimal, LPS-free conditions (Figure 3). At the highest amounts used $(250 \mathrm{ng} / \mathrm{mL}$ and 1 $\mu \mathrm{g}$ LPS $/ \mathrm{mL}$ ) the mode sizes were no different from the control and this may be due to a "rebound effect" where the highest response of the cells to these levels occurred before the 24-hour analysis end-point. However, it is the response of the cells to the low doses of endotoxin that is of particular interest as this data alone highlights the danger of using antibiotics (which may mask a low-level bacterial contamination) during clinical expansion of these cells. If the cells are reacting to LPS, present even at such low levels, then they are reacting to it in a way that may convert them from a beneficial anti-inflammatory phenotype to an inflammatory one, which is likely to hinder the outcomes of their use in the clinic.

Finally, we examined the effects of culturing the MSC1 line in reduced or sub-optimal levels of serum over a 24 hour period (Figure 4). In a similar manner seen with LPS titration there was a steady increase in the modal diameters of the EVs as the serum level decreased. This suggests that PTA can also serve as an indirect tool for detecting the effect of changes in the levels of nutrients present in the cell media. This observation also highlights that care must be taken when interpreting and comparing the EVs literature data from cells grown in low or no serum, since the PTA profiles of EVs derived from cells grown in such conditions are not the same.

\section{Conclusion}

We have demonstrated that PTA could offer a universal and nondestructive surrogate tool to monitor the health of MSCs in culture, simply by detecting changes in the EVs size profiles secreted by these cells upon exposure to extreme cases of non-ideal conditions and environmental factors, such as bacterial contamination, loss of nutrients or over-expansion.

\section{Conflicts of Interest}

There are no conflicts of interest to declare.

\section{Acknowledgements}

The work described in this paper was funded [in part] by the UK government Department for Business, Energy \& Industrial Strategy (BEIS)

\section{Notes}

Electronic Supplementary Information (ESI) available: cell doubling times, PTA mean diameter values of EVs derived from MSCs upon exposure to LPS.

\section{References}

1. Regulski MJ (2017) Mesenchymal Stem Cells: "Guardians of Inflammation". Wounds 29: 20-27. [Crossref]

2. Prockop DJ, Oh JY (2012) Mesenchymal stem/stromal cells (MSCs): role as guardians of inflammation. Mol Ther 20: 14-20.

3. Di Nicola M, Carlo-Stella C, Magni M, Milanesi M, Longoni PD, et al. (2002) Human bone marrow stromal cells suppress T-lymphocyte proliferation induced by cellular or nonspecific mitogenic stimuli. Blood 99: 3838-3843. [Crossref]

4. Krampera M, Glennie S, Dyson J, Scott D, Laylor R, et al. (2003) Bone marrow mesenchymal stem cells inhibit the response of naive and memory antigen-specific $\mathrm{T}$ cells to their cognate peptide. Blood 101: 3722-3729. [Crossref]

5. Galipeau J (2013) The mesenchymal stromal cells dilemma--does a negative phase III trial of random donor mesenchymal stromal cells in steroid-resistant graft-versus-host disease represent a death knell or a bump in the road? Cytotherapy 15: 2-8. Crossref]

6. von Bahr L, Sundberg B, Lönnies L, Sander B, Karbach H, et al. (2012) Long-term complications, immunologic effects, and role of passage for outcome in mesenchymal stromal cell therapy. Biol Blood Marrow Transplant 18: 557-564. [Crossref]

7. http://wwwfdagov/cvm/guidance/publishedhtml 
Morley GM (2019) Particle tracking analysis of extracellular vesicles as a non-destructive surrogate tool for mesenchymal stromal cell health monitoring

8. Lötvall J, Hill AF, Hochberg F, Buzás EI, Vizio DD, et al. (2014) Minimal experimental requirements for definition of extracellular vesicles and their functions: a position statement from the International Society for Extracellular Vesicles. J Extracell Vesicles 3: 26913

9. Börger V, Bremer M, Ferrer-Tur R, Gockeln L, Stambouli O, et al. (2017) Mesenchymal Stem/Stromal Cell-Derived Extracellular Vesicles and Their Potential as Novel Immunomodulatory Therapeutic Agents. Int J Mol Sci 18. [Crossref]

10. Li J, Lee Y, Johansson HJ, Mäger I (2015) Serum-free culture alters the quantity and protein composition of neuroblastoma-derived extracellular vesicles. J Extracell Vesicles 4: 26883. [Crossref]

11. R A Dragovic, C Gardiner, A S Brooks, D S Tannetta, D J Ferguson, et al. (2011) Sizing and phenotyping of cellular vesicles using Nanoparticle Tracking Analysis. Nanomedicine 7: 780 .
12. Saveyn H, De Baets B, Thas O, Hole P, Smith J, et al. (2010) Accurate particle size distribution determination by nanoparticle tracking analysis based on 2-D Brownian dynamics simulation. J Colloid Interface Sci 352: 593-600. [Crossref]

13. Hassan PA, Rana S, Verma G (2014) Making sense of Brownian motion: colloid characterization by dynamic light scattering. Langmuir 31: 3-12.

14. Waterman RS, Tomchuck SL, Henkle SL, Betancourt AM (2010) A new mesenchymal stem cell (MSC) paradigm: polarization into a pro-inflammatory $\mathrm{MSC} 1$ or an Immunosuppressive MSC2 phenotype. PLoS One 5: e10088. [Crossref].

15. J McFarland (1907) The Nephelometer: An Instrument for Estimating the Number of Bacteria in Suspensions Used for Calculating the Opsonic Index and for Vaccines. J Am Medical Association 49: 1176

Copyright: @2019 Morley GM. This is an open-access article distributed under the terms of the Creative Commons Attribution License, which permits unrestricted use, distribution, and reproduction in any medium, provided the original author and source are credited. 